\title{
TOWARDS AUTOMATED BIOIMAGE ANALYSIS: FROM FEATURES TO SEMANTICS
}

\author{
B. S. Manjunath, B. Sumengen, Z. Bi, J. Byun, M. El-Saban, D. Fedorov, N. Vu \\ Center for Bio-Image Informatics \\ Department of Electrical and Computer Engineering \\ University of California, Santa Barbara, CA 93106-9560 \\ http://www.bioimage.ucsb.edu
}

\begin{abstract}
Recent advances in bio-molecular imaging have afforded biologists a more thorough understanding of cellular functions in complex tissue structures. For example, high resolution fluorescence images of the retina reveal details about tissue restructuring during detachment experiments. Time sequence imagery of microtubules provides insight into subcellular dynamics in response to cancer treatment drugs. However, technological progress is accompanied by a rapid proliferation of image data. Traditional analysis methods, namely manual measurements and qualitative assessments, become time consuming and are often nonreproducible. Computer vision tools can efficiently analyze these vast amounts of data with promising results. This paper provides an overview of several challenges faced in bioimage processing and our recent progress in addressing these issues.
\end{abstract}

\section{INTRODUCTION}

While images are now a significant part of biology data, much of the image processing and analysis are still being done manually. This is partly attributed to the lack of robust image processing methods for bioimage applications. Automated image analysis methods not only help in reducing the manual labor but also facilitate tasks that are otherwise not feasible, such as mining large collections of images for quantitative features.

Typical steps in an automated analysis include extracting features at the low level, modeling the spatio-temporal variations in these features, and mining the feature/model space to discover interesting biological relationships. Each one of these steps pose significant computational challenges. In the context of microscope images, the low signal to noise ratio poses additional problems. Generally, in order to make progress in analysis of biological image data, there is a need to develop automated and robust methods that are adaptive to different imaging conditions.

In the following, we discuss our efforts at the Center for Bio-Image Informatics in developing such robust tools. The

This research is supported by NSF grant \#ITR-0331697.

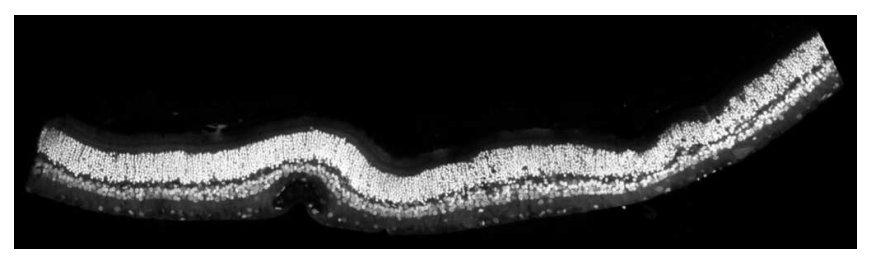

Fig. 1. Mosaic of 8 confocal 3D images of retina stained with TOPRO.

long term goal of our image informatics research is to facilitate quantitative analysis of bioimages and to develop a digital library wherein the users can browse through large bioimage collections, share their data, and perform computations on these data. Our current focus is on two specific data sets: 1) immunofluorescent images of retina cross sections and 2) time sequence imagery of microtubule dynamics. However, the methods and tools being developed are applicable to a broader range of images.

In the following, we briefly describe our recent work on automated registration (Section 2), segmentation and classification (Section 3), and tracking and modeling of microtubule dynamics (Section 4).

\section{REGISTRATION AND ENHANCEMENT}

Automated image registration is an important component in many bio-molecular image applications - from creating large field-of-view images to detecting and tracking changes. We developed a robust toolset for this task that is fully automated and easy to use. The basic problem of computing the correspondence between two or more images is achieved by a combination of feature extraction methods using condition number theory [1] and a RANSAC method for efficient pruning of candidate feature points [2]. Fig. 1 shows the mosaic of 8 confocal 3D cross section images of a retina stained with TOPRO.

The seamless mosaicking technique is also used in a new local image enhancement technique [3], which independently enhances image tiles before mosaicking them back together. 


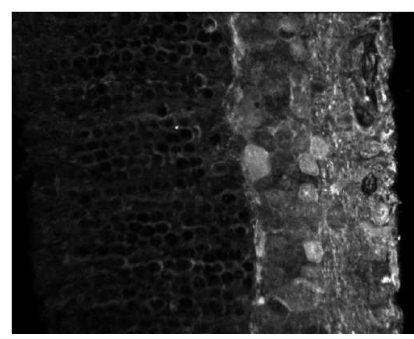

(a)

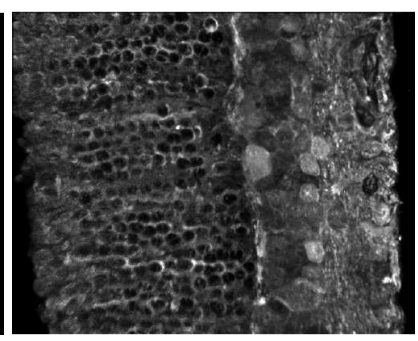

(b)
Fig. 2. (a) Original retina image. (b) Enhanced image.

This enhancement technique allows fast and accurate high dynamic range compression for display and correction of uneven intensities. Fig. 2 shows the spatial enhancement of a retinal image. For volume data, images can also be enhanced in the z-direction. Similarly this technique can be applied to time sequences of fluorescence images, e.g. microtubule videos where intensities are constantly diminishing due to photo bleaching.

\section{SEGMENTATION AND CLASSIFICATION}

Quantifying changes in morphological features or protein expressions is an important task. For example, researchers are interested in quantifying the redistribution of various proteins present before and after retinal detachment. In this context, we work extensively with immunofluorescence images of the retina captured during several stages of detachment and reattachment experiments. Fig. 3 shows example images of retina cross sections labeled with rod opsin antibody. The localization, intensity level, and spatial pattern of rod opsin protein expression are several essential features necessary to analyze this image set and must be reliably extracted.

To extract these features, we look at the intensity distribution around a neighborhood of each pixel. We represent these distributions by their means and standard deviations. Then by applying a recursive bi-partitioning using $\mathrm{k}$-means clustering, protein distributions can be classified into $k$ levels. Fig. 4 shows classification of protein distributions into four levels on two channels of a retina image. These quantized distributions of protein expressions are useful in modeling the retinal detachment process.

We also use texture features to characterize protein distributions. Our texture features are based upon Gabor filtering [4] and codeword creation [5]. Afterwards, each pixel is assigned a texture label, and then a histogram of pixel labels for the entire image is computed. These histograms can be thought of as high-dimensional feature vectors for the corresponding images. As an example, we look at the distributions of Glial Fibrillary Acid Protein (GFAP) from images of normal retinas and those after 1 day of detachment, 3 days of detachment, and 3 days of detachment followed by 28 days of reattachment. For visualization simplicity, we project the

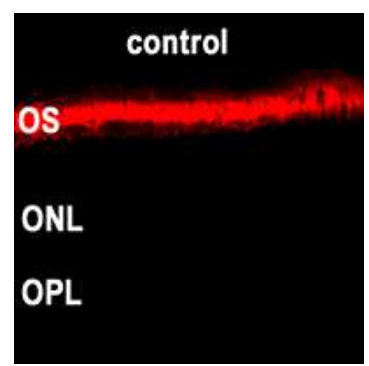

(a)

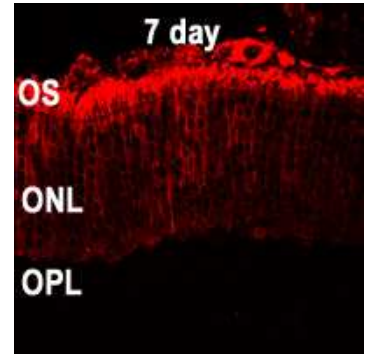

(c)

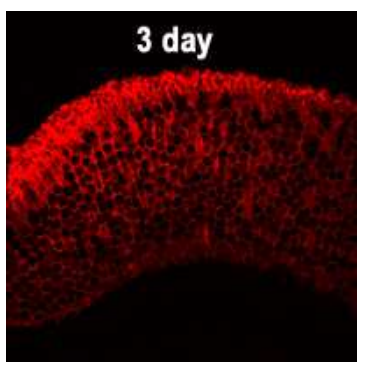

(b)

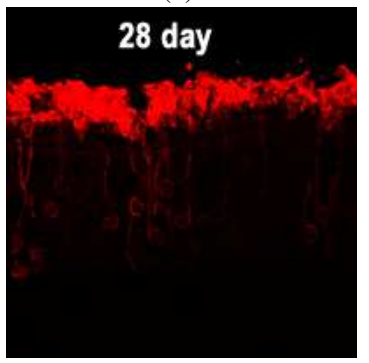

(d)
Fig. 3. Confocal images of retinas labeled with antibody to rod opsin (red). (A) Normal retina. (B) 3-day detached retina. (C) 7-day detached retina. (D) 28-day detached retina. (Best viewed in color)

data points from the original high dimensional feature space (we use histograms with 20 bins/clusters) to a two dimensional space spanned by the first two principle components (PC1 and PC2) and plot the distribution. Fig.5 shows that our feature vector and pixel clustering techniques provide good representation of protein distribution during various experimental stages. These quantitative results are helpful in evaluating how successfully the reattached tissue mimics the normal retina.

All vertebrate retinas are composed of three layers of nerve cell bodies and two layers of synapses (Fig. 3). Each layer has a different structure which consists of different cell bodies or synapses. The layer information is an important feature used to locate cells of interest and to measure the retinal function after an injury such as detachment. This information is also useful for narrowing search regions in a database and visualizing the query results, e.g. a query can focus on a particular layer. We developed a method for automatically assigning labels to immunofluorescence images of feline retina and a similarity search engine for these images based on several different criteria. In automatic layer classification, we found that Gabor texture feature is useful for coarse label assignments, and we are able to achieve about $76 \%$ accuracy in correctly labeling the different layers.

Nuclei detection is another important problem in bio-image analysis. For example, the number and density of photoreceptor nuclei within the outer nuclear layer (ONL) is directly correlated to visual function [6]. Images of nuclei usually show a slice of the nuclei in which the intensity distribution 


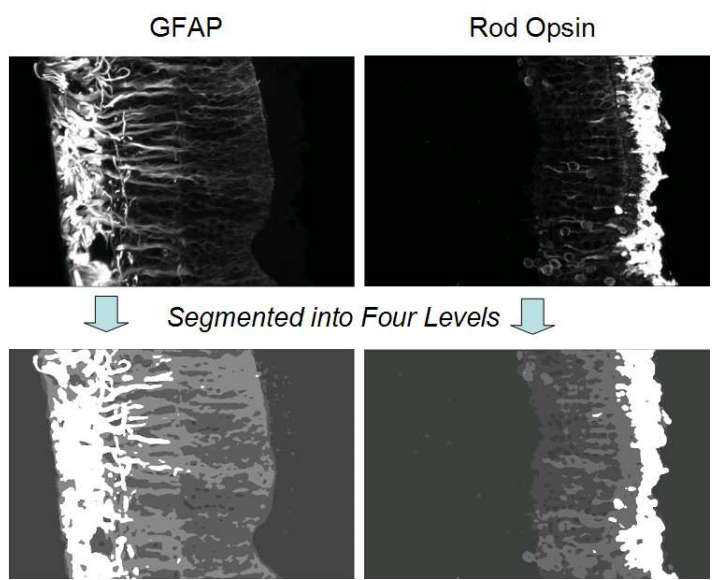

Fig. 4. Grouping of protein distributions into four levels.

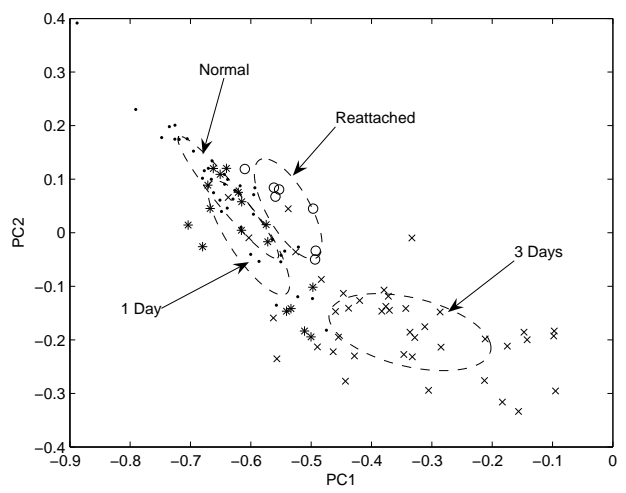

Fig. 5. First two principal components of texture features for GFAP under various experimental conditions. After 1 day of detachment, the features slightly depart from those under normal condition. After 3 days of detachment, texture features show clear distinction. Following reattachment, the GFAP distribution moves back toward the normal state.

of a nucleus can be modeled as a cylinder. We designed a nuclei detector that automatically detects cell bodies within the ONL. To evaluate the performance of the nuclei detector, the result is compared with the ground truth from 41 images. The automated nuclei detector approximates the number determined by manual counting with an average error of $\sim 4 \%$. Fig. 6 shows photoreceptor nuclei detection result in a confocal image of a normal retina. Refer to [7] for more details.

In addition to providing the cell count within the ONL, the detected nuclei are used to obtain measurements such as layer thickness and cell density profiles along the ONL. The ONL region is approximated using morphological operators based on the detected nuclei locations and photoreceptor size. A skeletal backbone or median of the resulting region is generated and used as a reference frame for subsequent measure-

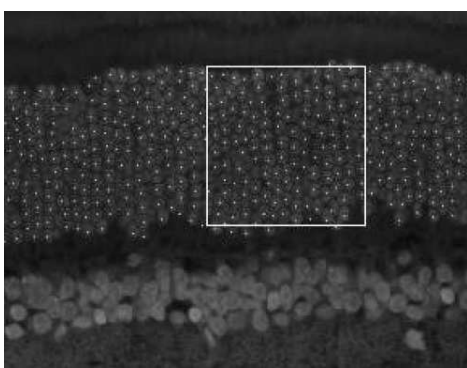

(a)

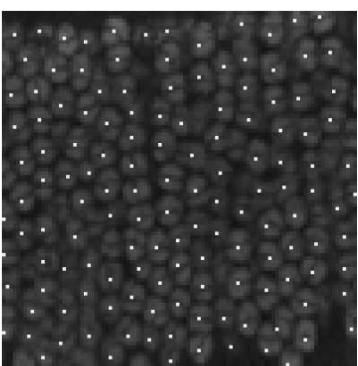

(b)
Fig. 6. Cell detection result, (a) detected cell centers, (b) magnified rectangular inset in (a).

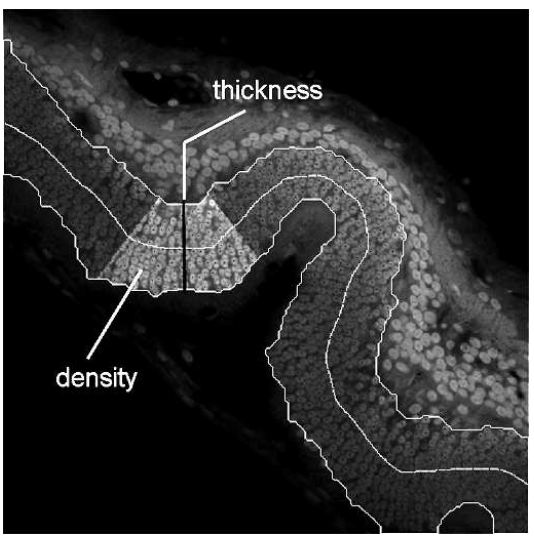

Fig. 7. ONL region approximated from detected nuclei and the thickness and density measurement at one backbone location.

ments. Then, the thickness profile is calculated as the width of the ONL along each backbone location. Similarly, the density profile is computed as the number nuclei inside a region surrounding each backbone location (highlighted region in Fig. 7) divided by the area of that region. Fig. 7 shows an example of the thickness and density analysis. Refer to [7] for more details.

\section{OBJECT DETECTION AND TRACKING}

The study of microtubule (MT) dynamics provides another interesting class of image sequence data analysis. MTs are cylindrical structures constituting one of the main components of the cytoskeleton [8]. MTs are highly dynamic polymers in the sense that their ends can be polymerizing or depolymerizing depending on need-a phenomenon called dynamic instability. This will have an effect of growing or shortening of the MTs. Currently, the imaging of MTs in a cell is carried out by high-resolution time-lapse microscopy leading to a time stack of fluorescence images. The dynamic behavior of MTs is affected by many factors, and abnormal dynamic behavior of the MTs can lead to cancer and neuro- 


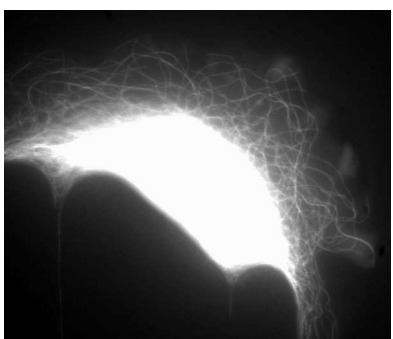

(a)

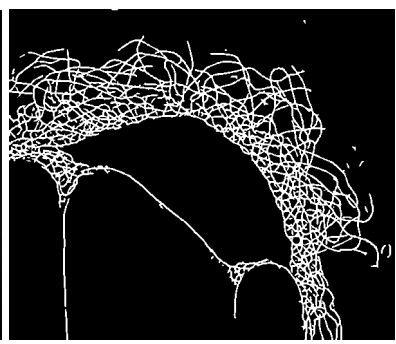

(b)
Fig. 8. a) First frame of a microtubule video. b) Detected microtubules.

degenerative diseases such as Alzheimer. To quantify the dynamic behavior of the MTs under different test subjects, biologists manually track the MT ends and generate life history plots of tube lengths for a relatively small number of MTs per stack-typically 5 to 10 . Manual tracking is both laborious and subjective, and results are difficult to replicate.

To improve MT analysis, we developed automated methods for the detection and tracking of MTs in order to estimate dynamic parameters. A filtering-based approach is used to detect the MT polymer mass. Oriented second derivative of Gaussian kernels matched to MT sizes and orientations reveal the MT structure while eliminating background noise, as shown in Fig. 8. Based on this MT binary mask, a morphological approach is then used to detect MT tips that are used in subsequent tracking. Using the detected tips in MT video, we developed a fully automated MT tip tracking method robust against occlusions and intersections. By solving the tracking problem for all the MTs and for the full time duration of the video simultaneously, we are able to resolve tracking conflicts and to overcome microtubule tip occlusion and mis-detection. Details of this approach are described in [9].

The MT detection and tracking results are then used in modeling the overall dynamic behavior of these MTs under various experimental conditions. For example, Hidden Markov Models can be used to obtain better insights into how experimental conditions affect the growth and shortening associated with the MTs, and data mining methods can be applied to identify frequent spatio-temporal patterns associated with the MT dynamics. For more details, we refer to [9].

\section{CONCLUSION}

Automated analysis of biological images is a very challenging task. Successful solutions require robust image processing, computer vision and data discovery tools that are designed with the understanding of the biological questions at hand. In this paper, we gave an overview of our recent work on analyzing retinal images and microtubule image sequences. We have developed an initial database of immunofluorescence images of vertebrate retina and microtubule videos [10] (see also http://www.bioimage.ucsb.edu/). The database provides methods for querying the raw images and the accompanying metadata and for combining the images with features for content-based retrieval and data mining. The long term vision of our digital library project is to create robust information processing methods for automated analysis of large collections of biomolecular image data that will advance our understanding of how cells respond to stress, injury, aging, disease, and treatment.

\section{ACKNOWLEDGMENTS}

We would like to thank professors Fisher, Feinstein, Wilson, and Dr. Verardo, Dr. Lewis, and Peck for many fruitful discussions relating to the bio-images and providing the data-sets used in our experiments.

\section{REFERENCES}

[1] C. S. Kenney et al., "A condition number for point matching with application to registration and postregistration error estimation," IEEE T. PAMI, vol. 25, no. 11, pp. 1437-1454, Nov 2003.

[2] M. Zuliani, C. S. Kenney, and B. S. Manjunath, "The multiransac algorithm and its application to detect planar homographies," in IEEE International Conference on Image Processing, Sep 2005.

[3] D. Fedorov, B. Sumengen, and B. S. Manjunath, "Tile-based framework for local enhancement of bio imagery," in CBII Tech. Rep., Nov. 2005.

[4] B. S. Manjunath and W.Y. Ma, "Texture features for browsing and retrieval of image data," IEEE T. PAMI, vol. 18, no. 8, pp. 837-42, Aug 1996.

[5] Wei-Ying Ma and B. S. Manjunath, "A texture thesaurus for browsing large aerial photographs," Journal of the American Society for Information Science, vol. 49, no. 7, pp. 633-48, May 1998.

[6] S. K. Fisher, G. P. Lewis, K. A. Linberg, and M. R. Verardo, "Cellular remodeling in mammalian retina: results from studies of experimental retinal detachment," Prog. in Retinal and Eye Research, vol. 24, pp. 395-431, 2002.

[7] J. Byun, N. Vu, B. Sumengen, and B. S. Manjunath, "Quantitative analysis of immunofluorescent retinal images," in IEEE ISBI 2006.

[8] B. Alberts et al., Essential Cell Biology: An Introduction to the Molecular Biology of the Cell, Garland Science, 1998.

[9] M. El-Saban et al, "Automated tracking and modeling of microtubule dynamics," in IEEE ISBI 2006.

[10] A. K. Singh, B.S. Manjunath, and R. F. Murphy, "A distributed database for biomolecular images," SIGMOD Record, vol. 33, no. 2, pp. 65-71, 2004. 